\title{
Comparison of SISO and MIMO Control Techniques for a Diagonally Dominant Vapor Compression System
}

\author{
Neera Jain, Member, IEEE, and Andrew G. Alleyne, Senior Member, IEEE
}

\begin{abstract}
For vapor compression cycle (VCC) systems, it is important to meet multiple control objectives in order to satisfy system capacity and efficiency requirements. Moreover, in the HVAC industry, it is generally preferred to tune multiple single-input-single-output (SISO) control loops rather than a single multiple-input-multiple-output (MIMO) control loop. This paper shows that when using an appropriate choice of feedback variables, a decentralized control approach consisting of individual SISO control loops performs as well as a MIMO control approach. An identified system model is shown to be diagonally dominant when using a decoupling set of inputoutput pairings. A linear quadratic Gaussian (LQG) control design is compared to a decentralized SISO control design through experimental results. Both controllers produce comparable time-domain performance characteristics.
\end{abstract}

\section{INTRODUCTION}

$\mathrm{I}_{\mathrm{t} t}^{\mathrm{N}}$ N vapor compression cycle (VCC) systems, it is important to meet multiple control objectives in order to satisfy system capacity and efficiency requirements. An idealized VCC system, as shown in Fig. 1, is a thermodynamic system driven by the phase characteristics of the refrigerant that is flowing through it. An ideal VCC system assumes isentropic compression, isenthalpic expansion, and isobaric evaporation and condensation.

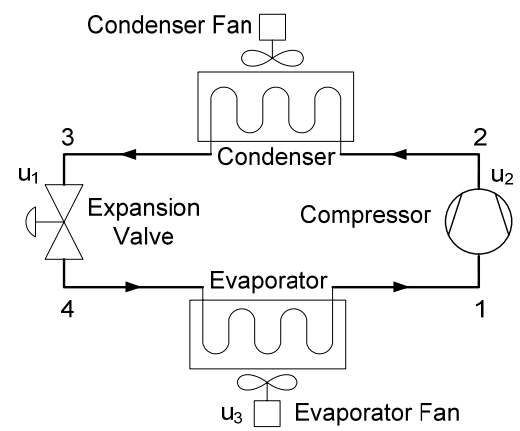

Fig. 1. Schematic of ideal subcritical VCC system.

The basic control objectives of a VCC system are to meet desired cooling capacity while maximizing system efficiency. These objectives can be conceptualized visually via Fig. 2 as discussed in [1]. The system coefficient of performance (COP), a measure of system efficiency, is proportional to the ratio between the enthalpy change across the evaporator coil and the enthalpy change across the

Neera Jain (njain2@illinois.edu) and Andrew G. Alleyne (alleyne@illinois.edu) are with the Mechanical Science and Engineering Department at the University of Illinois at Urbana-Champaign, Urbana, IL 61801 USA (phone: 217-244-9993; fax: 217-244-6534). compressor.

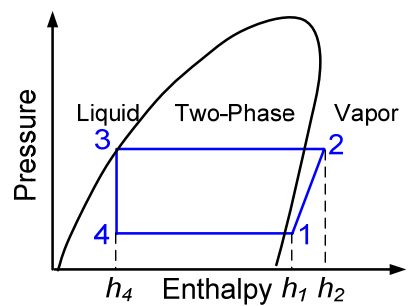

Fig. 2. P-h diagram for ideal subcritical VCC.

$$
C O P=\frac{|Q|}{W} \approx \frac{h_{4}-h_{1}}{h_{1}-h_{2}}
$$

Numerous control schemes have been developed with superheat and evaporation temperature (or pressure) as the feedback signals [2][3][4][5]. These references frequently noted the difficulty of controlling superheat and evaporation temperature with individual SISO control loops due to the physical coupling between these two feedback signals. It has been shown that multivariable control techniques [2][3][4] can be used to handle input-output (I/O) couplings while achieving desired performance objectives. However, for industrial practitioners and service engineers in the HVAC industry, it is generally preferred to tune multiple singleinput-single-output (SISO) control loops rather than a single multiple-input-multiple-output (MIMO) control loop.

In [1] a novel choice of output control variables was shown to effectively decouple system dynamics such that a decentralized control approach, consisting of individual SISO control loops, can be used to meet desired performance objectives. An extensive set of possible (I/O) pairings was considered, and the relative gain array (RGA) technique [6] was used to quantify the reduction in coupling for a given set of $\mathrm{I} / \mathrm{O}$ pairings. The final chosen set of decoupled I/O pairings is given in Table I. The baseline set represents current industry practice with respect to control of air-conditioning and refrigeration systems.

TABLE I

BASELINE AND DECOUPLED I/O PAIRINGS

\begin{tabular}{|l|l|l|}
\hline \multirow{2}{*}{$\begin{array}{l}\text { EEV opening } \\
(\%), u_{1}\end{array}$} & \multicolumn{1}{|c|}{ Control Output Parameter } \\
\cline { 2 - 3 } & Evaporator & \multicolumn{1}{c|}{ Decoupled } \\
\hline Compressor & Superheat, $T_{1-4}$ & Average System \\
Speed (rpm), $u_{2}$ & Pressure, $P_{(3+4) / 2}$ \\
\hline Evaporatorator & Differential \\
Power $(\%), u_{3}$ & Evaporator Air Inlet & Pressure, $P_{3-4}$ \\
\hline
\end{tabular}


Experimental results [1] confirmed that improved reference tracking was achievable when using a SISO control approach with this decoupled set of I/O pairings as compared to using the baseline set. Fig. 3 summarizes work completed in this research area thus far.

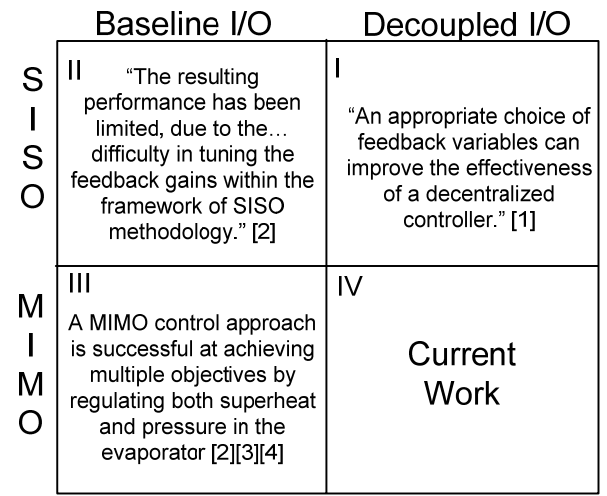

Fig. 3. Graphical summary of comparisons between SISO and MIMO control structures for VCC systems.

In this paper we extend the results of [1] to explore quadrant IV of Fig. 3. That is, we will show that when using these new I/O pairings, a decentralized control approach consisting of individual SISO control loops performs as well as a MIMO control approach. Section II describes the generation of identified models used to verify that the decoupled plant is diagonally dominant. Section III describes the SISO and MIMO control designs and presents simulation results. Finally, Section IV provides a comparison between experimental results for the SISO and MIMO controller implementations.

\section{SyStEM IDENTIFICATION AND ANALYSIS}

\section{A. System Model Identification}

While first principles models for VCC systems are available, Rasmussen [7] verified that system identification (ID) techniques can be used to construct accurate, loworder, local linear models of VCC systems. Therefore, in this paper, the dynamic response of a VCC is identified using a time domain system ID procedure. Three controllable inputs for a variable-speed VCC are considered: expansion valve opening, compressor speed, and evaporator fan speed (see $u_{1}$ through $u_{3}$ in Fig. 1). The condenser fan speed is not considered as a controllable input because in many cases, such as automotive systems, the condenser air flow rate is a function of vehicle speed and acts as a disturbance to the feedback loop.

The output responses to random Gaussian combinations of all three inputs (see Fig. 4) around a set of nominal operating conditions, were collected on an $\mathrm{A} / \mathrm{C}$ experimental test stand. For a more detailed description of the experimental system, see [8].
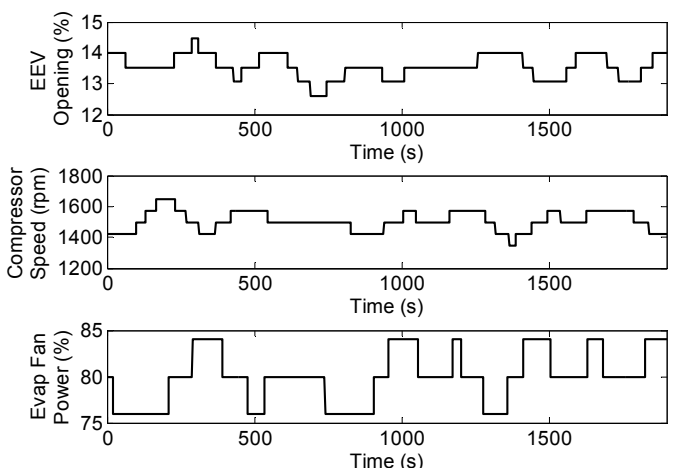

Fig. 4. Random Gaussian input signals for identification data.

A standard prediction error/maximum likelihood system ID algorithm in the System Identification Toolbox [9] was used to identify two third-order linear state space models using the baseline I/O pairings and the decoupled I/O pairings, respectively. Fig. 5 and Fig. 6 show the system identification results for each of the two models. The complete state space representation of each identified model is included in the Appendix. Note that in Fig. 6, evaporator pressure, rather than average system pressure, was used for the identification. The decoupled model representation shown in the Appendix was transformed appropriately such that $y_{1}$ represents average system pressure.
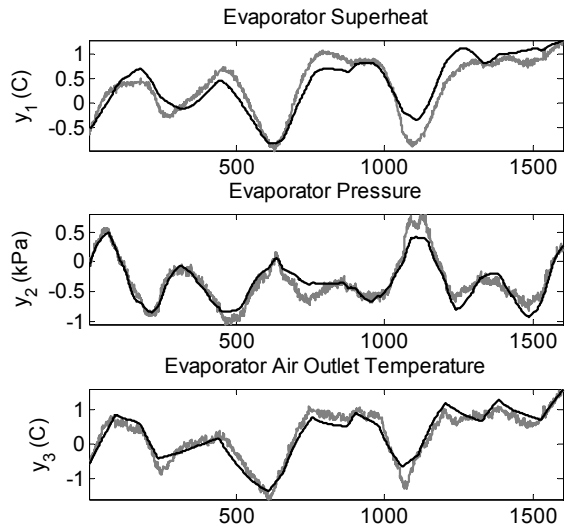

Fig. 5. Detrended system ID results using baseline $\mathrm{I} / \mathrm{O}$ pairings. $y_{1}:$ fit $=56.58 \% ; y_{2}$ : fit $=65.11 \% ; y_{3}:$ fit $=68.63 \%$.
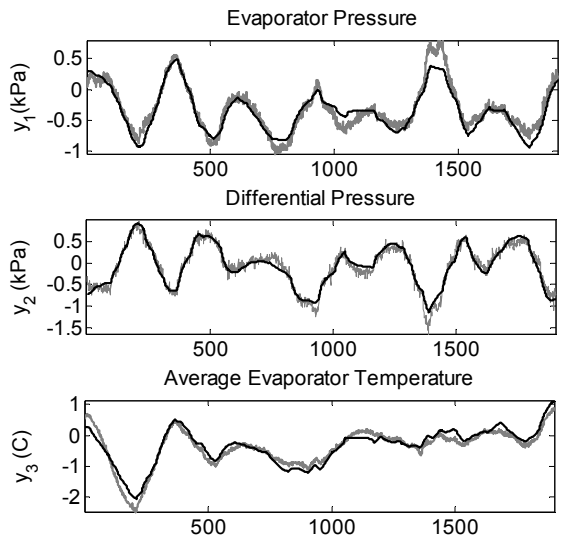

Fig. 6. Detrended system ID results using decoupled I/O pairings. $y_{1}:$ fit $=65.99 \% ; y_{2}:$ fit $=75.33 \% ; y_{3}:$ fit $=69.28 \%$. 


\section{B. Diagonal Dominance}

While the RGA number provided a metric for the reduction in coupling in [1], it is useful to analyze the diagonal dominance of the system which provides a more concrete measure of system decoupling. The interactions between the inputs and outputs of a MIMO system are characterized by the off-diagonal elements of the plant transfer function matrix, $G(\mathrm{~s})$, where

$$
G(s)=\frac{Y(s)}{U(s)}
$$

Normalizing these off-diagonal elements with respect to the diagonal ones results in (3)

$$
E \triangleq(G-\tilde{G}) \tilde{G}^{-1}
$$

where $\tilde{G}$ denotes a diagonal matrix containing the diagonal elements of $G(\mathrm{~s})$. "Generalized diagonal dominance" is defined as the ability of a system to be scaled such that it will be diagonally dominant [10] and is given by

$$
\mu(E)<1
$$

where $\mu$ is the structured singular value. The baseline model

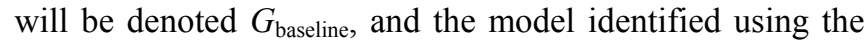
decoupled I/O pairings will be denoted $G_{\text {decoupled. }}$ The complete transfer function matrices for $G_{\text {baseline }}$ and $G_{\text {decoupled }}$ are given in the Appendix.

Fig. 7 shows $\mu(E)$ calculated for $G_{\text {baseline }}$ and $G_{\text {decoupled, }}$ for a frequency range of 0.001 to $6 \mathrm{rad} / \mathrm{s}$. Based on these results, we see that the decoupled plant model is indeed (generalized) diagonally dominant while the baseline plant model is not. This affirms that a decentralized SISO control approach can effectively be used on this VCC system when the decoupled I/O pairings are used. The key question remaining is whether a MIMO controller, for these types of systems, affords any performance benefits over a decoupled SISO controller design.

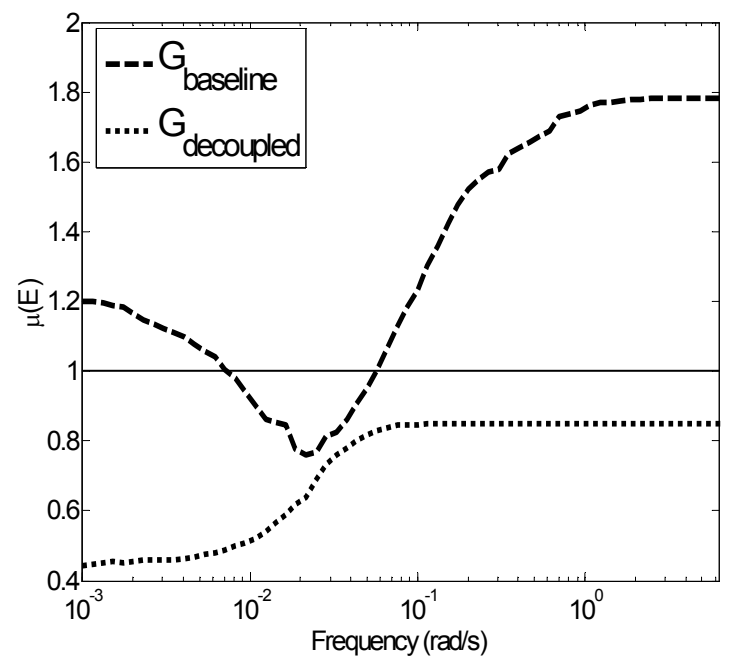

Fig. 7. Upper bound of structured singular value for $G_{\text {decoupled }}$ and $\mathrm{G}_{\text {baseline. }}$.

\section{CONTROLLER DESIGN}

\section{A. SISO Controller Design}

A decentralized control approach using three SISO control loops was used in [1] to effectively track step references (see Fig. 8).

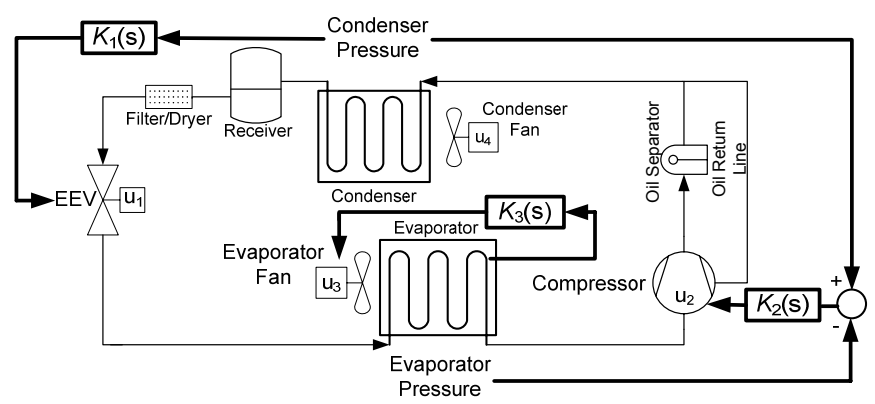

Fig. 8. A decentralized control approach for a VCC system using the decoupled I/O pairings.

A proportional-integral-derivative (PID) controller was used for each of the individual SISO control loops. The motivation for using this type of control structure was that it is widely used in the HVAC industry. Table II shows the chosen gains for each of the PID controllers, $K_{1}, K_{2}$, and $K_{3}$ $[1]$.

TABLE II

TUNED PID GAINS FOR INDIVIDUAL SISO CONTROLLERS

\begin{tabular}{|l|c|c|c|}
\hline \multicolumn{1}{|c|}{ Input/Controller } & $\begin{array}{c}\text { Proportional } \\
\text { Gain }\end{array}$ & $\begin{array}{c}\text { Integral } \\
\text { Gain }\end{array}$ & $\begin{array}{c}\text { Filtered } \\
\text { Derivative } \\
\text { Gain }\end{array}$ \\
\hline EEV Opening $u_{1} / K_{1}$ & 0.295 & 0.002 & 4 \\
\hline Compressor Speed $u_{2} / K_{2}$ & 17.5 & 0.55 & 3 \\
\hline Evap. Fan Speed $u_{3} / K_{3}$ & 2.84 & 0.028 & 60 \\
\hline
\end{tabular}

\section{B. MIMO Controller Design}

The desire to minimize the root-mean-square (RMS) error and control signals is motivated by a strong desire to minimize energy consumed during operation. This leads to the choice of a 2-norm based optimal controller rather than a worst case rejection (e.g. $H_{\infty}$ ) control design. Consequently, a linear quadratic Gaussian (LQG) [10] control approach is used in designing a MIMO controller for the experimental VCC system.

The original system is a Type 0 system and consequently can only track step reference inputs with a non-zero static error constant [11]. Therefore, the identified plant model $G_{\text {decoupled }}$ is augmented with three integrated error states to ensure zero steady-state error when the system is required to track step references. The schematic in Fig. 9 describes this augmented controller structure.

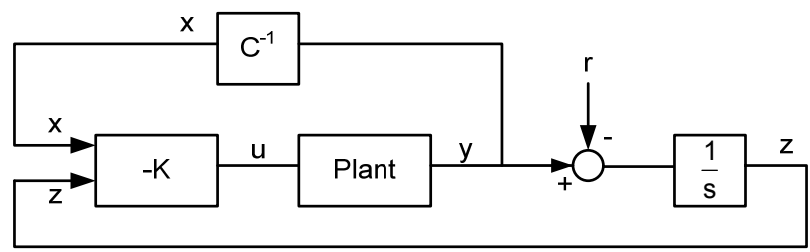

Fig. 9. Schematic of augmented LQG controller design. 


$$
\left[\begin{array}{c}
\dot{x} \\
\dot{z}
\end{array}\right]=\left[\begin{array}{cc}
A & 0 \\
C & 0
\end{array}\right]\left[\begin{array}{l}
x \\
z
\end{array}\right]+\left[\begin{array}{l}
B \\
0
\end{array}\right] u
$$

The weighting matrices, $Q$ and $R$, are chosen to obtain the desired controller performance and minimize $J$, the linear quadratic cost function.

$$
J=\int_{0}^{\infty}\left(\bar{x}^{T} Q \bar{x}+u^{T} R u\right) d t, \bar{x}=\left[\begin{array}{l}
x \\
z
\end{array}\right]
$$

The $R$ matrix is a diagonal matrix containing weights on the controller input, $u$.

$$
R=\left[\begin{array}{ccc}
100 & 0 & 0 \\
0 & 150 & 0 \\
0 & 0 & 100
\end{array}\right]
$$

The $Q$ weighting matrix is designed in terms of two block matrices, $K_{P}$ and $K_{I}$;

$$
Q=\left[\begin{array}{cc}
K_{P} & 0 \\
0 & K_{I}
\end{array}\right], K_{p}=(P C)^{T} P C
$$

$P$ is a diagonal matrix containing weightings on the original states, $x$, and $K_{I}$ is a diagonal matrix containing weightings on the integrated error states, $z$.

$$
K_{P}=\left[\begin{array}{ccc}
60 & 0 & 0 \\
0 & 50 & 0 \\
0 & 0 & 10
\end{array}\right] K_{I}=\left[\begin{array}{ccc}
10 & 0 & 0 \\
0 & 10 & 0 \\
0 & 0 & 1.5
\end{array}\right]
$$

Recall Eq. (1) which defines the COP of the system. The first and third inputs, expansion valve opening and evaporator fan speed, respectively, both affect the amount of heat removed from the air passing across the evaporator coil. The first diagonal entry in both the $K_{\mathrm{P}}$ and $K_{\mathrm{I}}$ matrices represents weightings on $u_{1}$ which is significantly larger than the last diagonal entry, representing weightings on $u_{3}$. This is because changing the average pressure $\left(P_{(3+4) / 2}\right)$ of the system has a greater effect on the COP than changing the average evaporator temperature $\left(T_{(1+4) / 2}\right)$.

The second input, compressor speed, affects the work required to operate the system. Consequently, $u_{2}$ is weighted most heavily in the $R$ matrix because it is the most expensive input with respect to its effect on the COP of the system (1). The weightings on $u_{2}$ in the $K_{\mathrm{P}}$ and $K_{\mathrm{I}}$ matrices are comparable to those on $u_{1}$. The MIMO controller was simulated using the identified plant model in Matlab Simulink.

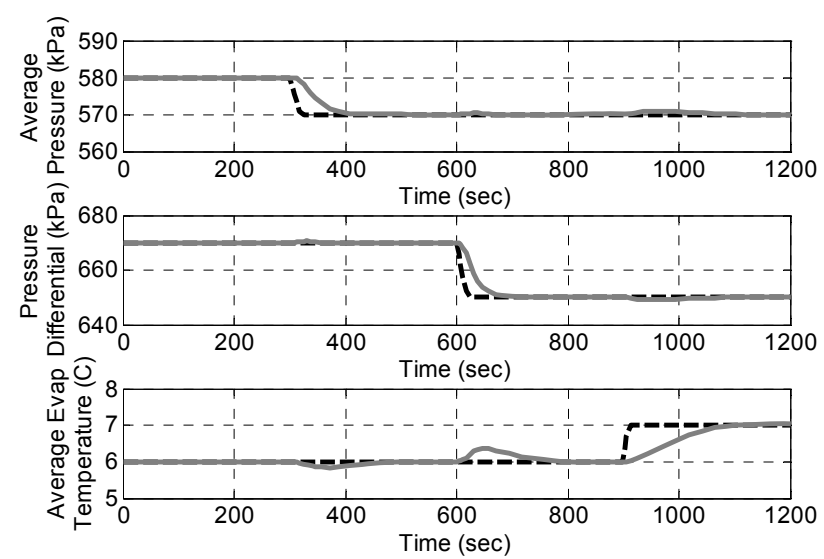

Fig. 10. Simulated reference tracking using MIMO controller. Reference signal $=$ '--', and output response signal $=$ '-'.
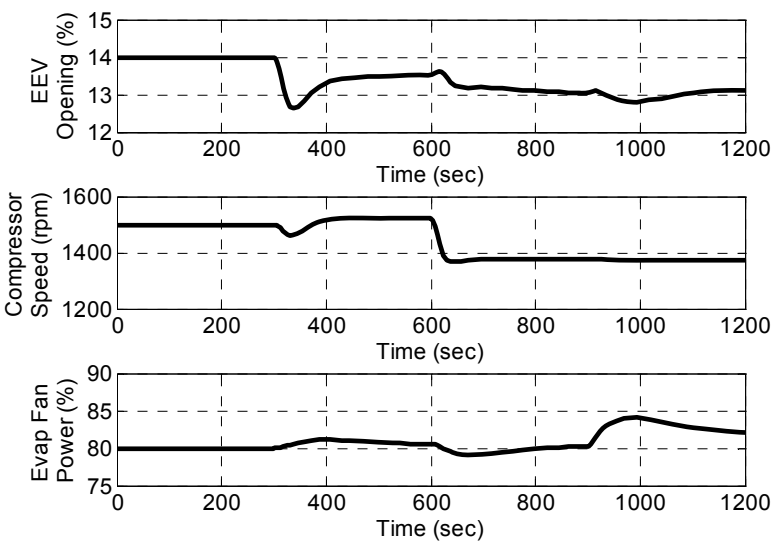

Fig. 11. Simulated actuation signals using MIMO controller.

\section{EXPERIMENTAL RESULTS}

The SISO and MIMO controller designs presented in the previous section were tested on the experimental system described in Section II. The reference tracking performance of each controller was tested by giving the system a series of step inputs around a nominal operating condition. Fig. 12 and Fig. 13 show the performance of the MIMO and SISO controllers, respectively.

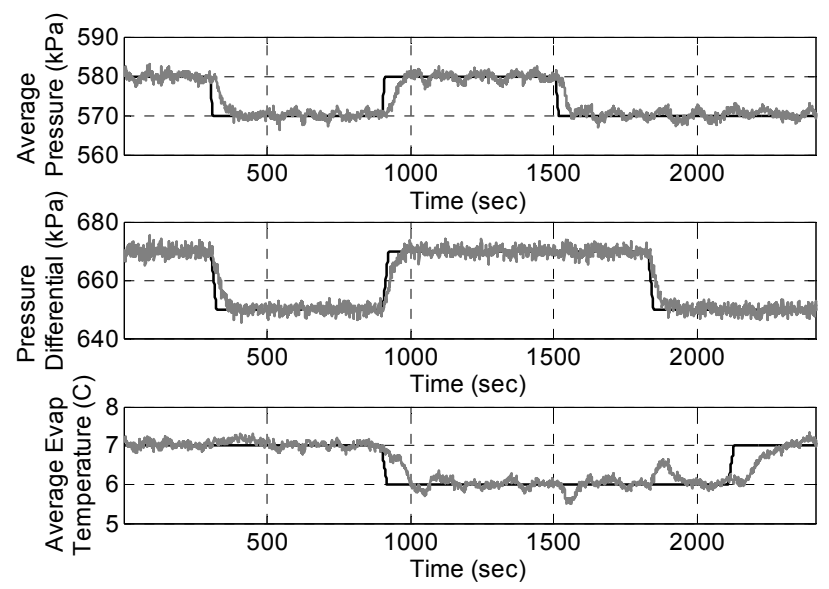

Fig. 12. Reference tracking results using MIMO controller. 

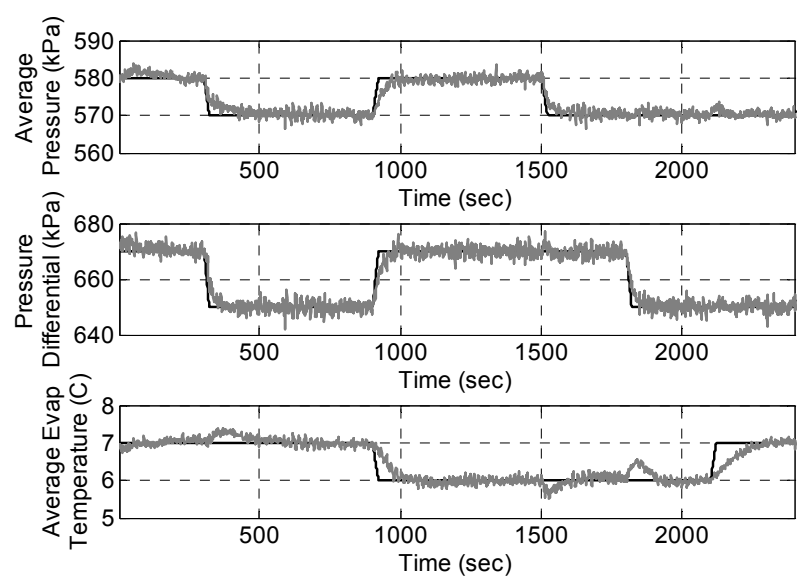

Fig. 13. Reference tracking results using SISO controller.

The MIMO controller tracks $P_{(3+4) / 2}$, the average pressure, and $P_{3-4}$, differential pressure, within \pm 3 and $\pm 4 \mathrm{kPa}$ of their desired set points, respectively, with a settling time of about 60 seconds. The average evaporator temperature, $T_{(1+4) / 2}$, is within $\pm 0.6^{\circ} \mathrm{C}$ of the desired set point throughout the test and has a settling time of about 150 seconds (see Fig. 12).

Using the SISO controller, the $P_{(3+4) / 2}$ controller, $K_{1}(s)$, and $P_{3-4}$ controller, $K_{2}(s)$, are able to track their respective reference signals within $\pm 4 \mathrm{kPa}$ and $\pm 5 \mathrm{kPa}$, respectively, with a settling time of about 50 seconds. The $T_{(1+4) / 2}$ feedback controller, $K_{3}(s)$, tracks the average temperature reference signal within $\pm 0.5^{\circ} \mathrm{C}$ error of the desired set point and with a settling time of about 100 seconds (see Fig. 13). While the time-domain performance characteristics are very similar between the two controllers, there are persistent oscillations in the average pressure signal when using the MIMO controller (see Fig. 12).

Another point of comparison is the ability of each controller to track individual outputs independently of the others. In Section II we showed that, with the decoupled I/O pairings, the system is diagonally dominant, implying independent tracking of set points should be achievable with both a SISO and MIMO controller.

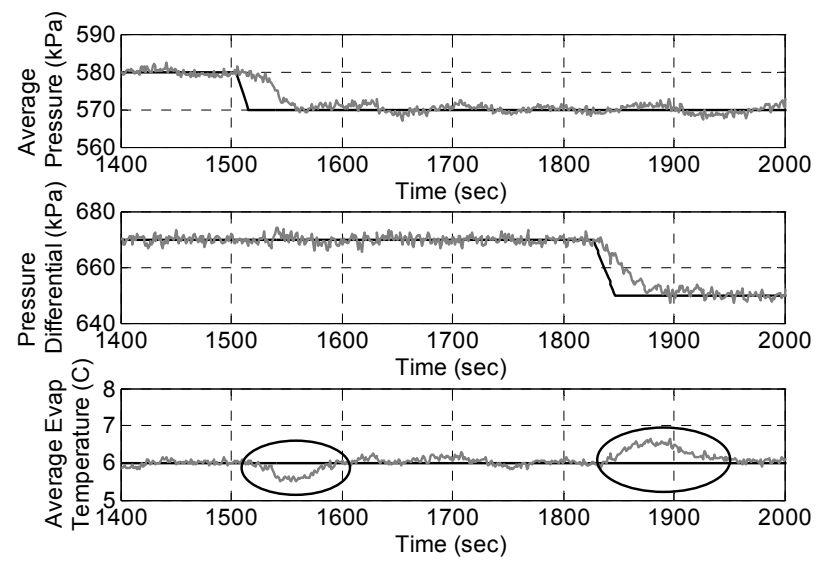

Fig. 14. Reference tracking with MIMO controller; zoomed in on $t=1400$ seconds to $t=2000$ seconds.

Fig. 14 and Fig. 15 provide a closer view of the time period $t=1400$ to $t=2000$ seconds. In both sets of experimental results, $T_{(1+4) / 2}$ is sensitive to step changes in both $P_{(3+4) / 2}$ and $P_{3-4}$ (see circled portions in Fig. 14 and Fig. 15). Similar behavior was seen in the simulation results for the MIMO controller (Fig. 10). At $t=1500$ seconds and $t=1800, T_{(1+4) / 2}$ deviates from the set point by $0.5^{\circ} \mathrm{C}$ with both the MIMO and SISO controllers whereas $T_{(1+4) / 2}$ otherwise tracks the set point within $\pm 0.2^{\circ} \mathrm{C}$ (again with both controllers).

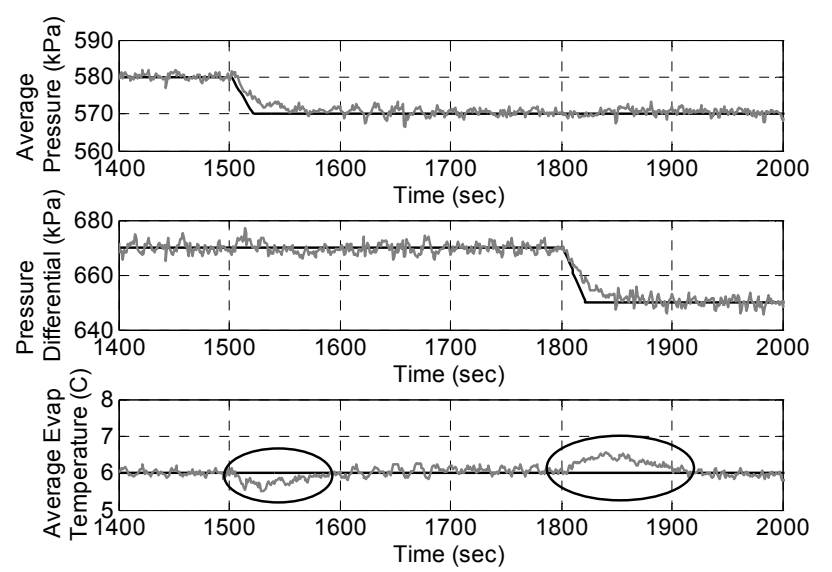

Fig. 15. Reference tracking with SISO controller; zoomed in on $t=1400$ seconds to $t=2000$ seconds.

\section{CONCLUSION}

This paper demonstrates that, for a VCC system in the HVAC industry, a decentralized SISO control structure can be as effective as a MIMO control structure when an appropriate choice of feedback variables is used. An LQG controller was designed and tested on an experimental VCC system, and the results were compared to a decentralized controller consisting of individual SISO loops. Both controllers produced comparable time-domain performance characteristics justifying the SISO feasibility.

\section{FUTURE WORK}

The analysis and controller design presented here is for a linear system representation while VCC systems are very nonlinear systems when operated over wide ranges of conditions. Any linear VCC system representation will vary greatly in its parameters about different operating points [12]. Consequently, future work must include a quantitative comparison between the SISO and MIMO controllers as well as an analysis of the performance and stability robustness of both controllers with respect to multiple operating conditions. Furthermore, by designing an outer optimization control loop, the benefits in performance achieved by using the decoupled I/O pairings can be fully leveraged to achieve desired control objectives.

\section{APPENDIX}

The identified state space $[\mathbf{A}, \mathbf{B}, \mathbf{C}, \mathbf{D}]$ system model for the baseline $\mathrm{I} / \mathrm{O}$ pairings is given in (10). 


$$
\begin{gathered}
A=\left[\begin{array}{ccc}
-0.00411 & 0.00392 & -0.00342 \\
0.00484 & -0.0221 & 0.00502 \\
0.0265 & -0.0209 & -0.0414
\end{array}\right] \\
B=\left[\begin{array}{ccc}
-0.000298 & -3.53 e-7 & 7.35 e-005 \\
0.00106 & -7.70 e-6 & 0.000326 \\
0.00291 & -3.12 e-5 & -0.000119
\end{array}\right] \\
C=\left[\begin{array}{ccc}
29.1 & -0.408 & 1.72 \\
-133 & 160 & 33.1 \\
15.3 & 6.07 & -2.31
\end{array}\right] \quad D=\left[\begin{array}{lll}
0 & 0 & 0 \\
0 & 0 & 0 \\
0 & 0 & 0
\end{array}\right] \\
\mathbf{u}=\left[\begin{array}{lll}
a_{v} & \omega_{c} & p_{f}
\end{array}\right]^{T} \mathbf{y}=\left[\begin{array}{lll}
P_{(1-4)} & P_{4} & T_{e, a i r}
\end{array}\right]
\end{gathered}
$$

The identified state space $[\mathbf{A}, \mathbf{B}, \mathbf{C}, \mathbf{D}]$ system model for the decoupled I/O pairings is given in (11).

$$
\begin{gathered}
A=\left[\begin{array}{ccc}
-0.00828 & -0.00512 & 0.00530 \\
-0.00480 & -0.00573 & -0.00411 \\
0.0135 & 0.0337 & -0.0451
\end{array}\right] \\
B=\left[\begin{array}{ccc}
0.000474 & -5.96 e-6 & 0.000157 \\
0.000544 & 1.54 e-6 & 5.85 e-6 \\
-0.00205 & 4.37 e-5 & -0.000281
\end{array}\right] \\
C=\left[\begin{array}{ccc}
88.7 & 228 & 4.66 \\
86.2 & 24.0 & 172 \\
23.2 & -0.949 & -1.09
\end{array}\right] \quad D=\left[\begin{array}{lll}
0 & 0 & 0 \\
0 & 0 & 0 \\
0 & 0 & 0
\end{array}\right] \\
\mathbf{u}=\left[\begin{array}{lll}
a_{v} & \omega_{c} & p_{f}
\end{array}\right]^{T} \mathbf{y}=\left[\begin{array}{lll}
P_{(3+4) / 2} & P_{3-4} & T_{(1+4) / 2}
\end{array}\right]
\end{gathered}
$$

The baseline plant transfer function matrix, $G_{\text {baseline }}(\mathrm{s})$, is given in (12). Similarly, the decoupled plant transfer function matrix, $G_{\text {decoupled }}(\mathrm{s})$, is given in (13).

\section{ACKNOWLEDGEMENT}

This work was supported in part by the sponsoring companies of the Air-Conditioning and Refrigeration Center at the University of Illinois at Urbana-Champaign.

\section{REFERENCES}

[1] Hencey, B., Jain, N., Li, B., and Alleyne, A., "Decentralized Feedback Structures of a Vapor Compression System," Proceedings of the 2008 ASME DSCC, Ann Arbor, MI, October 20-22, 2008.

[2] He, X. D., Liu, S., and Asada, H., "Multivariable Control of Vapor Compression Systems," HVAC\&R Research, vol. 4, pp. 205-230, 1998.

[3] Rasmussen, B. P., "Dynamic Modeling and Advanced Control of Air Conditioning and Refrigeration Systems," Phd. Dissertation, Dept. of Mechanical Engineering, University of Illinois at Urbana-Champaign, 2005.

[4] Shah, R., Rasmussen, B., Alleyne, A., "Application of Multivariable Adaptive Control to Automotive Air Conditioning Systems," International Journal of Adaptive Control and Signal Processing, Vol. 18, No. 2, pp. 199-221, March 2004.

[5] Keir, M. C., "Dynamic Modeling, Control, and Fault Detection in Vapor Compression Systems," MS Thesis, Dept. of Mechanical Engineering, University of Illinois at UrbanaChampaign, 2006.

[6] Bristol, E. H., "On a new measure of interaction for multivariable process control," IEEE Transactions on Automatic Control, vol. 11, no. 1, pp. 133-134.

[7] Rasmussen, B. P., Alleyne, A. G., and Musser, A. B., "Model-Driven System Identification of Transcritical Vapor Compression Systems," IEEE Transactions on Control Systems Technology, vol. 13, no.3, pp. 444-451.

[8] Keir, M. and Alleyne, A., "Feedback Structures for Vapor Compression Cycle Systems," 2007 American Control Conference, New York City, NY, pp. 5052-5058, July 11-13, 2007.

[9] Ljung, L., System Identification Toolbox: For Use with Matlab, The Math Works Inc., Natick, MA, 2001.

[10] Skogestad, S., and Postlethwaite, I., Multivariable Feedback Control, John Wiley \& Sons, New York, 1996.

[11] Nise, Norman S., Control Systems Engineering, John Wiley \& Sons, Inc., New Jersey, 2004.

[12] Rasmussen, B. and Alleyne, A., "Gain Scheduled Control of an Air Conditioning System Using the Youla Parameterization," 2006 American Control Conference, Minneapolis, MN, pp. 5336-5341, June 14-16, 2006.

$$
\begin{aligned}
& G_{\text {baseline }}(s)=\left[\begin{array}{ccccc}
\frac{-0.00411 s^{2}-0.000665 s-6.33 e-6}{s^{3}+0.0677 s^{2}+0.00136 s+4.55 e-6} & \frac{(-6.08 e-5) s^{2}+(6.25 e-7) s-1.56 e-8}{s^{3}+0.0677 s^{2}+0.00136 s+4.55 e-6} & \frac{0.00180 s^{2}+0.000165 s+4.61 e-6}{s^{3}+0.0677 s^{2}+0.00136 s+4.55 e-6} \\
\frac{0.306 s^{2}+0.0147 s+6.22 e-5}{s^{3}+0.0677 s^{2}+0.00136 s+4.55 e-6} & \frac{-0.00222 s^{2}-0.000111 s-3.90 e-7}{s^{3}+0.0677 s^{2}+0.00136 s+4.55 e-6} & \frac{0.0386 s^{2}+0.00123 s-2.47 e-6}{s^{3}+0.0677 s^{2}+0.00136 s+4.55 e-6} \\
\frac{-0.00486 s^{2}-0.000114 s-2.42 e-6}{s^{3}+0.0677 s^{2}+0.00136 s+4.55 e-6} & \frac{(2.00 e-5) s^{2}-(7.20 e-7) s-1.41 e-8}{s^{3}+0.0677 s^{2}+0.00136 s+4.55 e-6} & \frac{0.00338 s^{2}+0.000204 s+3.00 e-6}{s^{3}+0.0677 s^{2}+0.00136 s+4.55 e-6}
\end{array}\right] \\
& G_{\text {decoupled }}(s)=\left[\begin{array}{cccc}
\frac{0.157 s^{2}+0.00894 s+4.08 e-5}{s^{3}+0.0591 s^{2}+0.000722 s+2.34 e-6} & \frac{(2.54 e-5) s^{2}-(2.01 e-5) s-1.26 e-7}{s^{3}+0.0591 s^{2}+0.000722 s+2.34 e-6} & \frac{0.0140 s^{2}+0.000730 s-1.41 e-6}{s^{3}+0.0591 s^{2}+0.000722 s+2.34 e-6} \\
\frac{-0.299 s^{2}+0.00105 s+1.31 e-5}{s^{3}+0.0591 s^{2}+0.000722 s+2.34 e-6} & \frac{0.00703 s^{2}+(9.18 e-5) s+2.84 e-7}{s^{3}+0.0591 s^{2}+0.000722 s+2.34 e-6} & \frac{-0.0346 s^{2}+0.000300 s+3.58 e-7}{s^{3}+0.0591 s^{2}+0.000722 s+2.34 e-6} \\
\frac{0.0127 s^{2}+0.000214 s+1.08 e-6}{s^{3}+0.0591 s^{2}+0.000722 s+2.34 e-6} & \frac{-0.000187 s^{2}-(2.42 e-6) s-6.18 e-9}{s^{3}+0.0591 s^{2}+0.000722 s+2.34 e-6} & \frac{0.00395 s^{2}+0.000151 s+1.15 e-6}{s^{3}+0.0591 s^{2}+0.000722 s+2.34 e-6}
\end{array}\right]
\end{aligned}
$$

